\title{
Variations
}

Variations

Revue internationale de théorie critique

$22 \mid 2019$

Gorz, l'intempestif

\section{Cybercommunisme et capitalisme cognitif}

Sur quelques textes tardifs d'André Gorz

\section{Carlo Vercellone}

\section{(2) OpenEdition}

Journals

Édition électronique

URL : http://journals.openedition.org/variations/998

DOI : $10.4000 /$ variations. 998

ISSN : 1968-3960

Éditeur

Les amis de Variations

Référence électronique

Carlo Vercellone, "Cybercommunisme et capitalisme cognitif », Variations [En ligne], 22 | 2019, mis en ligne le 04 mars 2019, consulté le 19 avril 2019. URL : http://journals.openedition.org/variations/998 ; DOI : 10.4000/variations.998

Ce document a été généré automatiquement le 19 avril 2019

Les ami•e•s de Variations 


\title{
Cybercommunisme et capitalisme cognitif
}

\author{
Sur quelques textes tardifs d'André Gorz
}

Carlo Vercellone

\section{NOTE DE L'ÉDITEUR}

La réflexion d'André Gorz sur les mutations du capitalisme et l'émancipation du travail couvre plus d'un demi-siècle d'histoire. Il s'agit d'une pensée tourmentée et en perpétuel mouvement, bien qu'elle soit restée toujours fidèle à une méthode fondée sur deux éléments indissociables. D'un côté, le souci d'une analyse rigoureuse des contradictions immanentes du capitalisme expliquant pourquoi, pour paraphraser Marx, « la principale limite du capital est le capital lui-même ». De l'autre, la réflexion sur les conditions subjectives de son dépassement, car pour Gorz, la sortie du capitalisme doit être non seulement possible, mais aussi et surtout souhaitable, le produit d'un projet conscient d'émancipation, tout à la fois à l'échelle individuelle et collective.

À la différence du marxisme conventionnel et structuraliste, Gorz porte en fait son attention tout autant sur l'analyse des dispositifs de domination du capital que sur l'autonomie relative des sujets qui habitent les structures et peuvent les prendre comme les objets d'un processus de transformation. Il en résulte une lecture de l'histoire du capitalisme ouverte et ambiguë en ce sens qu'elle ne dispose d'un sens et d'une destination prédéterminée. C'est le fil conducteur de toute la réflexion que Gorz a mené sur la quête de sens des transformations du travail qui ont conduit du fordisme jusqu'à l'avènement du capitalisme cognitif. Une réflexion tourmentée dans laquelle Gorz semblera parfois douter de la possibilité de dépasser la rationalité économique du capital et de concilier les deux termes autour desquels a été historiquement posée au sein du marxisme la question de l'émancipation du salariat, à savoir : « l'émancipation du travail » et « l'émancipation dans le travail ». Par-delà l'attachement aux substrats philosophiques d'un marxisme existentialiste et humaniste qui met l'accent sur le 
caractère inextricable des rapports d'exploitation et d'aliénation, il est ainsi possible identifier trois principales étapes de la pensée de Gorz.

Une première étape est celle de l'âge du Fordisme et de sa crise, où Gorz s'érige en théoricien de l'autogestion et d'un projet d'émancipation qui associe étroitement émancipation du travail salarié et émancipation dans le travail. Une deuxième étape, débute avec Adieux au prolétariat (1980) et trouve son achèvement avec Métamorphoses du travail, Quête du sens (1988) et Capitalisme, socialisme, écologie (1991). Dans ce cadre la réflexion de Gorz, durant les années 1980 et jusqu'au milieu des années 1990, semble se résigner à un horizon où la « logique du capital » apparait comme un horizon indépassable. La rationalité économique du capital, du moins au niveau microéconomique, lui apparaît désormais comme la seule manière « économiquement rationnelle » de gérer la production dans la sphère du travail social de la nécessité. Dans ce cadre, la sortie du capitalisme prend alors la forme du projet d'une civilisation du temps libéré à réaliser grâce à une réduction drastique du temps de travail. La posture théorique et politique plus « réaliste » qu'il adopte durant cette période a sans doute contribué à rendre son message plus audible y compris au sein de vastes cercles du monde académique et des organisations de la gauche institutionnelle. C'est aussi la raison (implicite) pour laquelle nombre de ses exégètes la considèrent comme le point d'orgue de sa pensée. Cette interprétation est bien entendu parfaitement légitime : chacun a le droit de s'inspirer de la partie de la pensée d'un auteur ou d'une phase de sa vie durant laquelle s'est senti plus proche de lui.

Pourtant ce choix présente dans le cas de Gorz deux limites majeures. D'abord, il tend à oublier la manière dont les principales catégories et hypothèses employée par Gorz durant cette période seront profondément remises en question par Gorz lui-même durant la dernière période de sa vie intellectuelle. Ensuite, il occulte les raisons et l'importance du changement de trajectoire qui, depuis la fin des années 90 ont amené Gorz, dans ses derniers ouvrages $(1997,2003,2008)$, à adhérer simultanément à la proposition du revenu inconditionnel et à la thèse du capitalisme cognitif. Dans cette démarche, Gorz semble en fait entrevoir à nouveau la possibilité, sous des formes inédites, d'un projet de réappropriation des moyens de productions et d'émancipation dans le travail, un projet prenant la forme de ce qu'aujourd'hui nous appellerions les commons de l'écologie et de la connaissance.

C'est pourquoi en triant entre les belles feuilles de l'article de Carlo Vercellone consacré aux évolutions de la pensée de Gorz, à paraître dans l'ouvrage Matérialismes, culture et communication. Tome 3, économie politique de la culture, des médias et de la communication, dirigé par Fabien Granjon, Jacques Guyot et Christophe Magis, à paraître aux Presses des Mines à l'été 2019, nous avons privilégié les pages dédiées à cette dernière partie de sa vie.

\section{Les contradictions du capitalisme cognitif et l'économie de la connaissance}

1 Avec Misères du présent. Richesse du possible, puis L'immatériel et Ecologica, la réflexion de Gorz connaît un renouvellement important. Dans cette évolution, le dialogue qu'il instaure avec les analyses menées dans les revues Futur antérieur - en particulier, les contributions d'Antonio Negri et de Jean-Marie Vincent -, Alice et Multitudes sur les thématiques du general intellect, du travail immatériel et du capitalisme cognitif, joue un 
rôle considérable. En particulier, Gorz complète et modifie l'angle de son analyse des mutations du travail et de la crise de la loi de la valeur. A ce sujet, comme nous l'avons vu, son attention s'était, dans un premier temps, focalisée sur la robotisation de la production qui, en remplaçant le travail vivant par le travail mort, menait vers la crise de la société salariale. Une démarche dans laquelle, la référence au Fragment sur les machines des Grundrisse où Marx formule l'hypothèse du general intellect, était déjà mobilisée, mais d'une manière quelque peu tronquée. La crise de la loi de la valeur n'était appréhendée par Gorz que sous le seul angle de la science et de la technologie incorporées dans le capital constant. En revanche, le general intellect n'était pas pris en compte sur le plan des nouvelles qualités d'un savoir vivant du travail se concrétisant dans une intelligence collective.

2 Dans cette nouvelle étape donc, la réflexion de Gorz va octroyer une place centrale à la montée en puissance du rôle de la connaissance et de l'immatériel dans l'économie. Il finit aussi par renoncer à la notion trop floue et purement négative ${ }^{1}$ de post-fordisme pour inscrire sa réflexion autour de la problématique du capitalisme cognitif. Par ce concept, nous désignons le passage du capitalisme industriel à une nouvelle phase du capitalisme «dans laquelle la dimension cognitive et intellectuelle du travail devient dominante et l'enjeu central de la valorisation du capital et des formes de la propriété porte directement sur la transformation de la connaissance en une marchandise fictive " (Vercellone 2008: 72). Le capitalisme cognitif se présente ainsi comme une tentative de sortie de la crise du capitalisme industriel, mais aussi comme une restructuration plus profonde du capitalisme face aux défis posés par une économie fondée sur la connaissance. Cette restructuration vise à enserrer à l'intérieur de sa logique les nouvelles forces productives issues de la société du general intellect, tout en étouffant son potentiel d'émancipation (Vercellone, 2013b). Gorz apporte une contribution essentielle à la caractérisation du capitalisme cognitif en formulant la thèse selon laquelle celui-ci " n'est pas un capitalisme en crise, il est la crise du capitalisme qui ébranle la société dans ses profondeurs (Gorz 2003 : 81-82). Il faut entendre par là, la contradiction de plus en plus aiguë qui se creuse entre la logique rentière du capitalisme cognitif et celle immanente à une économie fondée sur le savoir qui " contient [...] en son fond une négation de l'économie capitaliste marchande » (Gorz 2003: 76)². Cette contradiction se manifeste tant sur le plan du rapport capital/travail dans la sphère de la production que sur celui d'un divorce croissant entre la logique de la valeur et celle de la richesse.

\section{La déconnexion entre valeur et richesse, et l'essor d'une économie de rente}

3 Une première difficulté structurelle que rencontre le capitalisme cognitif, plonge ses racines dans le statut même de bien commun de la connaissance (Gorz, 2003; 2008), à savoir dans son caractère non rival, non contrôlable et cumulatif ${ }^{3}$. À la différence des biens matériels, la connaissance ne se détruit pas dans la consommation. Mieux encore, elle s'enrichit lorsqu'elle circule librement entre les individus. Chaque nouvelle connaissance engendre une autre connaissance selon un processus cumulatif. C'est pourquoi l'appropriation privative de la connaissance n'est réalisable qu'au moyen de l'établissement de barrières artificielles à l'accès. La tentative de transformer la connaissance en une marchandise fictive engendre ainsi, selon Gorz (2003) une situation paradoxale dans laquelle plus la valeur d'échange de la connaissance augmente 
artificiellement, plus sa valeur d'usage, c'est à dire son utilité, baisse, du fait même de sa privatisation et de sa raréfaction. De manière plus générale, pour nombre de productions intensives en connaissances, comme, par exemple les logiciels mais aussi les médicaments et bien d'autres biens matériels, les temps de travail, et donc les coûts de reproduction sont très faibles, et dans certains cas tendent vers zéro. Ces biens devraient donc être vendus à des prix très faibles, voire parfois être cédés gratuitement, ce qui risquerait de conduire à la diminution drastique de la valeur monétaire de la production et donc des profits qui lui sont associés. Pour contrer cette tendance, l'enjeu clé pour le capital devient alors une stratégie visant à créer des rentes de position monopoliste. La réalisation de cet objectif, dans lequel le profit se mue en rente ${ }^{4}$, est poursuivie de deux manières complémentaires.

4 La première consiste à renforcer la composante immatérielle, souvent purement symbolique et superflue de la marchandise, au moyen du design, du marketing et de l'image de marque. En effet, "Les qualités immatérielles incomparables procurent à la firme productrice l'équivalent d'un monopole et la possibilité de s'assurer une rente de nouveauté, de rareté, d'exclusivité. Cette rente masque, compense et souvent surcompense la diminution de la valeur au sens économique que la baisse des coûts de production entraîne pour les produits en tant que marchandises par essence échangeables entre elles selon leur rapport d'équivalence » (Gorz, 2008 : 38). La seconde stratégie repose sur un processus de renforcement et d'extension des droits de propriété intellectuelle (copyright, brevets) poussé jusqu'à remettre en cause les frontières mêmes entre découverte et invention en provoquant ce que l'on a appelé la tragédie des " anticommons» de la connaissance. Dans le deux cas, le résultat est le même. Le capital dans la tentative de survivre à la crise de la loi de la valeur et de perpétuer de manière forcée la primauté de la valeur d'échange développe toujours davantage des mécanismes de raréfaction de l'offre permettant de bâtir artificiellement une rareté des ressources. «Ce déguisement - poursuit Gorz - ne change cependant rien à la réalité de bien commun du bien ainsi déguisée " (Gorz, 2008: 37). La connaissance, les idées, l'immatériel, finalement tout ce qui est convertissable en langage numérique :

« reste une non-marchandise non vendable dont l'accès et l'usage libres sont interdits parce qu'ils demeurent toujours possibles, parce que le guettent les "copies illicites", les "imitations", les usages interdits. Le soi-disant propriétaire lui-même ne peut les vendre c'est-à-dire en transférer la propriété privée à un autre, comme il le ferait pour une vraie marchandise ; il ne peut vendre qu'un droit d'accès ou d'usage "sous licence". L'économie de la connaissance se donne ainsi pour base une richesse ayant vocation d'être un bien commun, et les brevets et copyrights censés le privatiser n'y changent rien ; l'aire de la gratuité s'étend irrésistiblement. L'informatique et internet minent le règne de la marchandise à sa base » (Gorz, $2008: 37)$.

5 Il existe ainsi, entre capitalisme cognitif et économie de la connaissance, une situation de radicale non correspondance entre rapports sociaux de production et forces productives. Elle se manifeste à travers une "déconnexion patente entre valeur et richesse", qui illustre de manière exemplaire «la crise du capitalisme dans ses fondements épistémiques » (Gorz, 2004 : 214). Pour bien comprendre le sens de cette affirmation, il faut rappeler comment, pour Marx, le concept de valeur se différencie de celui de richesse pour deux raisons fondamentales qui sont sans doute biens présentes à l'esprit de Gorz, même s'il ne les explicite pas. La première raison concerne la manière dont la valeur (des marchandises) dépend des difficultés de la production et donc du temps de travail, tandis que la richesse, elle, dépend de la valeur d'usage, de l'abondance et, en dernière instance, 
de la gratuité. Or, comme nous l'avons vu, la logique capitaliste du capitalisme industriel avait sans doute trouvé, à cet égard, une sorte de légitimité historique grâce à sa capacité de développer la richesse en produisant toujours davantage de marchandises avec moins de travail et donc à prix de plus en plus faibles, en permettant de satisfaire une quantité croissante de besoins. Dans le capitalisme cognitif, cette liaison dynamique entre valeur et richesse est pourtant rompue, voire elle s'inverse : c'est en fait le maintien artificiel de l'emprise du royaume de la marchandise et de la propriété privée qui engendre la rareté et empêche le passage vers une société fondée "sur la gratuité et l'abondance ». La seconde raison renvoie à une précision importante que Marx (1992) explicite dans la Critique du Programme de Gotha, lorsqu'il affirme qu'en réalité, "Le travail n'est pas la source de toute richesse. La nature est tout autant source des valeurs d'usage (qui sont bien, tout de même, la richesse réelle) » (Marx, 1992 : 49). Ce passage de Marx souvent cité comme l'expression d'une conception non productiviste et écologique du rapport d'interaction entre homme et nature, permet de saisir une autre implication importante du diagnostic gorzien relatif au divorce entre valeur et richesse qui ébranlerait le capitalisme cognitif dans ses fondements épistémiques. Il s'agit de la manière dont le potentiel de négativité du capitalisme cognitif ne consiste qu'à rendre artificiellement rares des ressources abondantes et gratuites. Il se concrétise aussi, selon Gorz, dans l'accélération de la course à la prédation des ressources naturelles non renouvelables. En fait le capitalisme cognitif ne supprime pas le productivisme propre au capitalisme industriel. Il le réarticule, il le renforce, dans le cadre de l'alliance historique scellée par le capital et la science (Gorz, 2003), qui met les nouvelles technologies au service d'une quête de standardisation et de transformation marchande du vivant ${ }^{5}$.

6 Cette tendance accentue le risque de destruction de la biodiversité et de déstabilisation écologique de la planète. Plus encore, la rencontre entre révolution numérique et sciences de la vie, impulse, selon Gorz, la transition vers une « civilisation posthumaine " dans laquelle les conditions les plus essentielles de la reproduction du genre humain seront remodelées et produites comme des marchandises. Mais, à la fin des comptes, conclut Gorz, si «le capital et la science se servent l'une de l'autre dans leurs buts respectifs» (Gorz, 2003 : 120), «l'abolition de la nature a pour moteur non le projet démiurgique de la science, mais le projet du capital de substituer aux richesses premières, que la nature offre gratuitement et qui sont accessibles à tous, des richesses artificielles et marchandes: transformer le monde en marchandise dont le capital monopolise la production, se posant ainsi en maitre de la vie» (Gorz, 2003 : 119). Dans ce cadre, «le divorce entre valeur et richesse [...] apparaît maintenant lié au divorce entre connaissance et savoir vécus » (Gorz, $2003: 110)$. En somme, « le capitalisme cognitif est le mode sur lequel le capitalisme se perpétue quand ses catégories ont perdu leur pertinence [...] Virtuellement dépassé, le capitalisme se perpétue en employant une ressource abondante - l'intelligence humaine - à produire de la rareté. Y compris de la rareté d'intelligence» (Gorz, 2003: 81-82). En ce sens aussi, l'un des enjeux clé de la bifurcation historique actuelle nous renvoie, selon Gorz, à la capacité d'opposer au projet du capitalisme cognitif celui d'une "société de l'intelligence " capable de réconcilier connaissance et expérience sensible pour aboutir vers ce qu'il appelle « une science plus qualitative » (Gorz, 2004) fondée sur un paradigme holistique des processus de la nature. 


\section{Le travail immatériel entre autonomie potentielle et servitude volontaire}

7 La crise de la loi de la valeur et la perte de pertinence des catégories de l'économie politique classique du capitalisme industriel se manifeste aussi sur le plan du rapport capital/travail. De ce point de vue, le changement clé est la montée en puissance du travail cognitif et l'autonomie potentielle dont il dispose dans l'organisation sociale de la production, en rupture avec le modèle taylorien. Le travail cognitif se présente en effet comme la combinaison complexe d'un travail intellectuel de réflexion, de communication, de partage, d'élaboration du savoir, qui s'effectue tant en amont, que dans le cadre même du travail immédiat de production dans l'entreprise (Gorz, 1997). Dans ce cadre, le travail mesuré au temps passé et certifié dans l'entreprise n'est le plus souvent qu'une fraction du temps effectif de travail. La centralité du travail matériel, mesuré en unité de travail abstrait simple, non qualifié, cède la place à celle d'un travail immatériel auquel ni les mécanismes tayloriens de prescription du travail ni « les étalons de mesure classiques, ne sont désormais applicables» (Gorz, 2003: 11). En effet, «l'hétérogénéité des activités de travail dites cognitives, des produits immatériels qu'elles créent et des capacités et des savoirs qu'elles impliquent, rend non mesurables tant la valeur des forces de travail que celles de leurs produits» (Gorz, 2003: 34). Et, poursuit Gorz, «la crise de la mesure du travail entraîne inévitablement la crise de la mesure de la valeur ", (2003: 34), en déstabilisant le système d'équivalence qui règle les échanges marchands sur la base d'une norme qui gravite autour du temps de travail socialement nécessaire à leur production. Mais ce qui est le plus important est la manière dont la crise de la mesure du travail est étroitement associée au caractère non prescriptible du travail cognitif et donc à un affaiblissement du contrôle du capital sur la force de travail. En fait, tout semble se passer comme si « les capacités constitutives de la force de travail ne sont plus subsumables par le capital en tant qu'entité distincte, et que la nature de la production requiert maintenant un mode de coopération sociale autoorganisée, susceptible de déboucher tôt ou tard sur l'émancipation individuelle et collective des travailleurs » (Gorz, 2004 : 207). En somme, ce n'est plus le savoir incorporé au capital fixe, mais " l'intelligence collective » qui se présente désormais comme la principale force productive. Plus fondamentalement encore, dans une économie fondée sur la connaissance, ce n'est plus dans les entreprises mais dans la société que s'opère une partie essentielle du processus de création de savoirs et de richesses, selon une logique qui trouve une illustration exemplaire dans le modèle de coopération horizontale et non marchande du logiciel libre.

8 Dans ce cadre, la valorisation du capital repose de plus en plus sur la prédation de richesses et la mise au travail des facultés des travailleurs produites à l'extérieur du système économique des entreprises, dans ce que Gorz appelait auparavant la sphère du temps libre et du travail pour soi. La dichotomie entre un univers du travail hétéronome et de la nécessité, d'une part et un univers du travail autonome à but non économique, d'autre part, vole en éclat. Elle cède la place à la tension nouvelle entre deux modèles économiques et sociaux alternatifs qui traversent et englobent d'emblé ces deux sphères ayant définitivement perdu leur étanchéité. La synergie entre l'intelligence collective et les moyens d'autoproduction high tech rend en fait de nouveau concevable la perspective d'une réappropriation collective du savoir et des forces productives qui pointe « vers un 
avenir où pratiquement tout le nécessaire et le désirable pourront être produit dans des ateliers coopératifs ou communaux » (Gorz, 2008 : 40-41). Nous avons là, selon Gorz, une situation dualiste d'une nature complétement inédite et qui recèle à la fois toutes les misères du présent et toute la richesse du possible du capitalisme cognitif. La « richesse du possible » parce que l'intelligence collective est porteuse d'une « économie première faite d'activités, d'échanges et de relations non marchandes» (Gorz 2003 :11). Une autre économie qui entretient avec le capital une relation complexe de domination et de conflits. Elle se nourrit de pratiques d'exode du travail salarié qui à la faveur même de la crise pourraient progressivement conduire à la rendre hégémonique. En effet, ce sont toutes les formes de pouvoir que le capital avait établi sur le savoir et la division du travail que «la révolution informationnelle commence de fissurer » (Gorz, 2008:31) en rendant « la mégamachine industrielle virtuellement obsolète " (Gorz, 2008: 40). Gorz renoue ainsi, sur des bases nouvelles, avec un projet autogestionnaire qui trouve son incarnation principale dans ce que nous appellerons aujourd'hui les communs de l'écologie et du numérique. Certes, il (2008) précise bien que ces transformations radicales ne sont qu'une possibilité et que rien ne garanti qu'elles se réaliseront. La remise en cause même des frontières jadis établies entre les sphères de la nécessité et de la liberté peut aller dans deux directions opposées : celle de la poursuite d'un exode de la société salariale édifiant les formes d'organisation alternatives de l'essor d'un postcapitalisme; celle d'une subordination de plus en plus accrue de l'ensemble des temps de vie et de la subjectivité du travail au capital. C'est sur le plan de cette alternative que s'inscrit le terrain de conflit central entre capital et travail à l'âge du capitalisme cognitif et d'une crise écologique de plus en plus profonde.

9 Selon Gorz, « la lutte engagée entre les logiciels propriétaires et les logiciels libres [...] a été le coup d'envoi du conflit central de l'époque. Il s'étend et se prolonge dans la lutte contre la marchandisation de richesses premières - la terre, les semences, les savoirs et compétences communs, constitutifs de la culture du quotidien et qui sont les préalables de l'existence d'une société» (Gorz, 2008: 39). De la tournure que prendra cet affrontement entre deux modèles alternatifs, va dépendre le mode de sortie, civilisée ou barbare, d'un capitalisme qui de toute manière ne pourrait pas survivre à la catastrophe écologique. Toutefois son issue reste dramatiquement incertaine. La richesse du possible est en fait, étroitement entremêlée aux misères du présent. Aucun automatisme n'assure le passage de l'autonomie formelle à l'autonomie réelle de la force de travail. Au contraire, le travail cognitif va souvent de pair avec des formes nouvelles d'exploitation et d'aliénation encore plus redoutables que celles connues sous le taylorisme dans lequel subsistait une frontière assez nette entre le temps du travail salarié et le temps libre. Le travail cognitif suppose, en fait, une implication de toute la personne, une mobilisation au service de l'entreprise de l'ensemble des temps sociaux et des savoirs qui proviennent justement de ce travail de production de soi réalisé par les individus dans le temps dit " libre ». Le capitalisme cognitif empiète en somme de plus en plus sur la vie elle-même. Le modèle taylorien de la prescription de tâches cède ainsi la place à celui de la " prescription de la subjectivité ", où l'enjeu central devient le contrôle total du temps et de l'esprit des salariés. Il s'agit d'obtenir des salariés l'intériorisation des objectifs définis par la direction et, plus encore, de façonner un mode de penser qui conduit effectivement le travailleur à se concevoir comme un entrepreneur de soi-même. Les formes classiques de l'exploitation peuvent alors s'associer à des dispositifs prenant « l'apparence de l'autoexploitation ou de la servitude volontaire " (Gorz 2004 : 206). Dans ce cadre, les formes classiques de l'exploitation peuvent alors s'associer et prendre l'apparence de l'auto- 
exploitation et de la servitude volontaire. C'est pourquoi, dans le capitalisme cognitif, la production de subjectivité devient plus que jamais un terrain de conflit central. C'est aussi pourquoi, davantage encore qu'à l'âge du fordisme, les questions de la maîtrise du temps devient, selon Gorz, de plus en plus un enjeu central pour penser un projet d'émancipation du travail des formes d'aliénation et d'exploitation propres au capitalisme cognitif.

\section{Revenu d'existence et développement de l'économie des communs}

10 L'une des conséquences les plus significatives de cette évolution de la pensée de Gorz est le changement de sa position à l'égard de la proposition d'un revenu social garanti (RSG) inconditionnel et indépendant de l'emploi, proposition à laquelle il s'était jusqu'au milieu des années 1990 opposé, en lui préférant un projet de réduction progressive, à l'échelle sociale, de la dépense en travail-emploi effectuée dans la sphère de la nécessité. Contrairement à des lectures à notre sens hâtives, la justification de ce revirement ne se trouve point dans une adhésion abrupte aux thèses de la «fin du travail ». Gorz, à l'inverse, précise clairement que diagnostiquer la crise du travail-emploi est toute autre chose que parler d'une crise du travail en soi, entendue comme l'éventail de possibilités où un travail émancipé aurait la possibilité de s'épanouir. C'est ce qu'il affirme sans ambiguïté aucune, par exemple, dans un entretien avec Denis Clerc de 1998 :

\footnotetext{
"Je tiens moi aussi que le travail ne peut jamais ni disparaître ni manquer si on prend "travail" au sens anthropologique de transformation et appropriation de la nature. Je dis que ce qui, en revanche, tend à disparaître, c'est la forme emploi du travail : c'est-à-dire le travail en tant qu'il nous est donné à faire par un employeur qui nous paie pour cela. Quand je dis que "le volume de l'emploi se contracte", je ne parle donc pas [...] du travail sous ses diverses formes, ni du nombre des emplois. Je parle de ce qu'il est convenu d'appeler le volume de travail qui a la forme emploi, volume mesuré dans toutes les statistiques en nombre d'heures par année " (Gorz, $1998 b$ : 62).
}

11 Écartée la thèse de la fin du travail, deux arguments principaux plaident alors, selon Gorz, pour la proposition d'un revenu d'existence entièrement déconnecté de l'emploi. Le premier est que lorsque « l'intelligence et l'imagination (le general intellect) deviennent la principale force productive, le temps cesse d'être la mesure du travail ; de plus, il cesse d'être mesurable » (Gorz 1997 : 140) Il en résulte qu'il devient de moins en moins possible d'envisager une réduction homogène du temps de travail en définissant « une quantité de travail incompressible à accomplir par chacun au cours d'une période déterminée » (Gorz 1997 : 140). Le second argument est que le caractère inconditionnel d'un RSG permettrait de renforcer la liberté effective de choix de la force de travail, tout en libérant le potentiel de développement de ce mode de production alternatif tant au public qu'au marché que nous appelons aujourd'hui les communs de l'écologie et de la connaissance. Dans cette démarche, Gorz lui-même indique à plusieurs reprises l'importance d'une définition du RSG en tant que revenu primaire. Son fondement, de manière semblable à l'approche que nous défendons avec Jean-Marie Monnier (2007), devrait reposer sur la remise en cause de « l'assimilation abusive du concept de travail et du concept de travail-emploi » et donc, sur l'affirmation «que le travail peut être improductif de capital, mais producteur de richesses non marchandes et donner donc droit à un revenu versé en dehors de toute logique comptable de mesure de l'effort individuel et de sa nature» (Vercellone, 2013: 
143). Il en est ainsi, par exemple, lorsque Gorz affirme que « la revendication de la garantie inconditionnelle d'un revenu suffisant doit surtout... signifier d'emblée que le travail dépendant n'est plus le seul mode de création de richesse, ni le seul type d'activité dont la valeur sociale doit être reconnue » (2003: 100). Autrement dit, poursuit-il, « la garantie d'un revenu suffisant doit marquer l'importance croissante, virtuellement prépondérante, de cette autre économie créatrice de richesses intrinsèques, ni mesurables, ni échangeables. Elle doit marquer la rupture entre création de richesse et création de valeur ». Et de préciser: " elle doit mettre justement en évidence que " chômage » ne signifie ni inactivité sociale ni inutilité sociale, mais seulement inutilité à la valorisation directe du capital » (2003: 100). Avec une grande capacité d'anticipation par rapport au débat actuel, il semble aussi associer cette caractérisation du RSG comme revenu primaire à celle qui en fait une institution du commun en affirmant qu'il correspond « en fin de compte, à la mise en commun d'une partie de ce qui est produit en commun, sciemment ou non ${ }^{6} »($ Gorz, $2003: 101)$.

Dans ses derniers essais, Gorz nous livre une interprétation saisissante et rigoureusement marxienne de l'acuité des contradictions objectives et subjectives du capitalisme actuel. La transition du capitalisme industriel au capitalisme cognitif aurait marqué une rupture par rapport au mode de fonctionnement normal du capitalisme qui s'exprime à travers la crise de la loi de la valeur. Elle va de pair avec l'épuisement des deux leviers sur lesquels le capitalisme industriel était parvenu jadis à asseoir une sorte d'hégémonie effective, au sens gramscien de ce terme, à savoir la capacité d'incarner " la vision du futur sur laquelle une civilisation règle ses projets, fonde ses buts idéaux, ses espérances » (Gorz, 1988 : 22). D'une part, l'expansion du royaume de la marchandise n'est en fait plus en aucune manière synonyme d'une expansion parallèle de la richesse sociale. La déconnexion patente entre valeur et richesse manifeste l'impuissance du capitalisme contemporain, dans les pays d'ancienne industrialisation, à développer encore les forces productives comme un instrument de lutte contre la rareté, en réalisant ce que Marx, à tort ou à raison, jugeait être le rôle historique du capital : la création des pré-conditions favorisant le passage du royaume de la nécessité au royaume de la liberté. Plus encore, la perpétuation de sa logique en ferait désormais un facteur de raréfaction artificielle de ressources, une pure force de destruction de l'environnement et de l'équilibre écologique de la planète. D'autre part, l'essor d'une intelligence collective et l'autonomie du travail cognitif remettent radicalement en cause le rôle démiurgique du capital, sa prétention à se présenter comme une condition indispensable de l'organisation de la production et de l'innovation. Cette évolution ouvre sur un nouvel horizon du rapport antagoniste capital/ travail dont l'enjeu central porte sur la maîtrise même du développement des forces productive et de l'innovation technologique. Le capitalisme serait ainsi parvenu «dans son développement des forces productives à une frontière, passée laquelle il ne peut tirer pleinement partie de ses potentialités qu'en se dépassant vers une autre économie. L'acteur potentiel de ce dépassement est le "capital humain", pour autant qu'il a tendance à s'émanciper du capital » (Gorz, $2003:$ 84).

14 Nous avons ici, dans l'analyse du dernier Gorz, une approche située aux antipodes des interprétations de son héritage nous invitant à radicaliser, en son nom, «la critique du marxisme» (Caillé, 2013: 189). Au contraire, sur tous les points de l'analyse qu'il développe dans ses derniers écrits, qu'il s'agisse de la crise de la loi de la valeur, de la proposition de RSG, de l'autonomie du travail cognitif et du rôle de l'intelligence 
collective comme principale force productive, nous pouvons plutôt constater une forte proximité avec le programme de recherche néo-marxiste du courant opéraiste ${ }^{7}$. Gorz n'a pas pu suivre le débat intense qui durant la dernière décennie s'est ouvert sur le concept de Commun, pensé comme un «mode de production » alternatif tout autant à la logique bureaucratique de l'État qu'à celle de l'économie capitaliste de marché. Toutefois, nombre des passages contenus dans ces derniers écrits ébauchent un horizon semblable de la sortie du capitalisme et nous pensons qu'il partagerait aujourd'hui en grande partie cette perspective. Pour conclure, notre espoir est que cette introduction et hommage à la pensée de Gorz puisse valoir aussi d'encouragement à une nouvelle génération de chercheurs à prolonger son analyse, pour aller avec Marx, au-delà du Marx et du capitalisme comme fin de l'histoire.

\section{BIBLIOGRAPHIE}

Clerc (Denis), « Les Trois vies d'André Gorz », Alternatives Économiques, n² 263, 2007, p. 80-82.

Caillé (Alain), « Au-delà du marxisme et du capitalisme », in Fourel (Christophe), Caillé (Alain) dir. Sortir du capitalisme. Le scénario Gorz, Lormont, Le Bord de l’Eau, 2013, p. 189-198.

Gorz, (André), Adieux au prolétariat. Au-delà du socialisme, Paris, Galilée, 1980.

Gorz, (André), Métamorphoses du travail. Quête du sens - Critique de la raison économique, Paris, Galilée, 1988.

Gorz, (André), Capitalisme, socialisme, écologie, Paris, Galilée, 1991.

Gorz, (André), Misères du présent. Richesse du possible, Paris, Galilée, 1997.

Gorz, (André), « Le travail perd de sa centralité dans la vie les gens », entretien avec Denis Clerc, Alternatives économiques, $\mathrm{n}^{\circ}$ 157, 1988, p.62, https://www.alternatives-economiques.fr/travailperd-de-centralite-vie-gens/00018678

Gorz, (André), L'immatériel : connaissance, valeur et capital, Paris, Galilée, 2003.

Gorz, (André), "Économie de la connaissance et exploitation des savoirs », entretien avec Yann Moulier-Boutang et Carlo Vercellone, Multitudes, $n^{\circ}$ 15, 2004, p. 205-216.

Gorz, (André), Ecologica, Paris, Galilée, 2008.

Monnier (Jean-Marie), Vercellone (Carlo), Fondements et faisabilité du revenu social garanti », Multitudes, $\mathrm{n}^{\circ}$ 27, Janvier, 2007, p. 73-84.

Marx (Karl), Le Capital, Livre I, Paris, PUF, 1993.

Moulier-Botang (Yann), Capitalisme cognitif, Paris, Amsterdam, 2007.

Vercellone, (Carlo), « La thèse du capitalisme cognitif. Une mise en perspective historique et théorique», in Colletis (Gabriel), Paulré (Bernard) coord., Les nouveaux horizons du capitalisme, Paris, Economica, Vercellone, (Carlo), 2008, p. 71-95. 
Vercellone, (Carlo), «The Crisis of the Law of Value and the Becoming-Rent of Profit », in Fumagalli (Andrea), Mezzadra (Sandro) eds., Crisis in the Global Economy : Financial Markets, Social Struggles, and New Political Scenarios, New York, Semiotext, 2010, p. 85-118.

Vercellone, (Carlo), « Capitalisme cognitif et revenu social garanti comme revenu primaire », in Fourel (Christophe), Caillé (Alain) dir. Sortir du capitalisme. Le scénario Gorz, Lormont, Le Bord de l'Eau, 2013, p. 189-198.

Vercellone, (Carlo), « From the Mass Worker to Cognitive Labour: Historical and Theoretical Considerations ", in van der Linden (Marcel), Roth (Karl Heinz) eds., Beyond Marx, Londres, Brill, 2013b, p. 417-443.

\section{NOTES}

1. Négative car le terme post-fordisme ne faisait que définir le nouveau capitalisme par ce qu'il n'était plus, plutôt que par ce qu'il était devenu et contenait de complétement nouveau.

2. Une interprétation que nous partagions et diamétralement opposée à celle de MoulierBoutang. Selon ce dernier la principale entrave au développement du capitalisme cognitif se trouverait dans la résistance du capitalisme industriel et non dans les contradictions internes au capitalisme cognitif, notamment celles inhérentes à son rapport avec les forces productives d'une économie fondée sur la connaissance (Moulier-Boutang, 2007 : 152).

3. Notons que le recours à la notion de «bien commun » employée ici par Gorz serait considéré comme impropre par la théorie économique conventionnelle. Dans la typologique des biens proposés par la théorie néoclassique, le caractère non rival, difficilement excluable et cumulatif de la connaissance en fait plutôt ce qu'on appelle un bien public ou collectif (imparfait) correspondant à un cas de défaillance du marché.

4. Le rôle croissant des rentes de monopole souligné par Gorz fait partie de ce que, dans les approches néo-opéraiste nous appelons la tendance au devenir rente du profit. Ce concept donne à la logique rentière du capitalisme cognitif une signification plus large que chez Gorz. Il désigne non seulement la croissance exponentielle de formes rentières "classiques", tels les droits de propriété intellectuelle ou la rente financière et immobilière. Il désigne aussi la manière dont le capital productif lui-même ne remplit le plus souvent, sur le plan de la connaissance, aucune fonction nécessaire pour la mise en place d'une coopération du travail cognitif qui n'a plus besoin de la fonction de direction du capital. En ce sens, le profit d'entreprise lui-même, comme jadis la rente du propriétaire foncier, repose sur une situation où la capture de la plus-value s'opère à partir d'une position d'extériorité par rapport à l'organisation de la production. Marx lui-même avait envisagé une évolution semblable dans le Livre III du Capital (Vercellone, 2008 ; 2010).

5. L'alliance entre Science et Capital, selon Gorz, trouverait son support épistémologique dans l'utopie scientiste dont s'est nourrie dès le départ la "révolution scientifique " : celle d'une pensée libérée de la " prison du corps » et, finalement, d'une reproduction de l'espèce humaine débarrassée du pouvoir de la fonction de l'utérus de la femme. Elle aurait trouvé aujourd'hui un soubresaut dans la conception des pionniers de l'intelligence artificielle et des théoriciens de l'ectogenèse. Les premiers en poursuivant l'idéal d'un univers mécanique de la pensée d'où serait banni tout ce qui relève de l'expérience sensible du monde vécu et échapperait aux techniques de calcul mathématique. Les seconds en poussant « la haine méprisante de la nature et de la naturalité de la vie » dans la voie d'une industrialisation et "d'une rationalisation technoscientifique de la reproduction humaine » (Gorz, 2003 : 117).

6. Toutefois, dans d'autres passages, Gorz finit par « rejeter » cette fondation du revenu social garanti comme « rémunération d'une activité productive » en affirmant que cette 
formulation resterait accrochée à la valeur-travail et, en prenant acte de la mise au travail totale de la personne, finirait par la légitimer (Gorz, $2003: 30$ ). Il opte alors pour une justification « non économique mais politique » selon laquelle le revenu n'est plus compris comme la rémunération d'une création de richesse, mais comme « ce qui doit rendre possible le déploiement d'activités qui sont une richesse et une fin pour ellesmêmes » (Gorz, 2003 : 103). Ce glissement de la justification du revenu garanti pose plusieurs problèmes dont il n'est malheureusement pas possible de discuter dans le cadre de cet article (Vercellone, 2013).

7. Sans oublier, sur d'autres plans, l'influence du groupe Krisis et des théoriciens de la critique de la valeur (Jappe, Kurz, Postone).

INDEX

Mots-clés : capitalisme cognitif, économie de la connaissance, commons, revenu d'existence, André Gorz 